\title{
A Posteriori Error Estimator for Mixed Approximation of the Navier-Stokes Equations with the $c_{a b c}$ Boundary Condition
}

\author{
J. EL Mekkaoui, M A. Bennani, A.Elkhalfi \\ Mechanical engineering laboratory \\ Faculty of sciences and techniques-B.P. 2202 Route Imouzzer \\ Fes
}

\begin{abstract}
In this paper, we introduce the Navier-Stokes equations with a new boundary condition. In this context, we show the existence and uniqueness of the solution of the weak formulation associated with the proposed problem. To solve this latter, we use the discretization by mixed finite element method. In addition, two types of a posteriori error indicator are introduced and are shown to give global error estimates that are equivalent to the true error. In order to evaluate the performance of the method, the numerical results are compared with some previously published works and with others coming from commercial code like ADINA system.
\end{abstract}

\section{Keywords-Navier-Stokes Equations; $C_{a, b, c}$ boundary} condition; Mixed Finite element method; Residual Error Estimator;

\section{INTRODUCTION}

This paper describes a numerical solutions of Navier-stoks equations with a new boundary condition generalizes the will known basis conditions, especially the Dirichlet and the Neumann conditions. So, we prove that the weak formulation of the proposed modelling has an unique solution. To calculate this latter, we use the discretization by mixed finite element method. Moreover, we propose two types of a posteriori error indicator which are shown to give global error estimates that are equivalent to the true error. To compare our solution with the some previously ones, as ADINA system, some numerical results are shown. This method is structured as a standalone package for studying discretization algorithms for PDEs and for exploring and developing algorithms in numerical linear and nonlinear algebra for solving the associated discrete systems. It can also be used as a pedagogical tool for studying these issues, or more elementary ones such as the properties of Krylov subspace iterative methods [15].

The latter two PDEs constitute the basis for computational modeling of the flow of an incompressible Newtonian fluid. For the equations, we offer a choice of two-dimensional domains on which the problem can be posed, along with boundary conditions and other aspects of the problem, and a choice of finite element discretizations on a quadrilateral element mesh.

Whereas the discrete Navier-Stokes equations require a method such as the generalized minimum residual method (GMRES), which is designed for non symmetric systems [15].

\author{
A. Elakkad \\ Department of mathematics \\ Regional Centre for Professions of Education and Training, \\ Fes, B.P: 243 Sefrou Morocco
}

The key for fast solution lies in the choice of effective preconditioning strategies. The package offers a range of options, including algebraic methods such as incomplete LU factorizations, as well as more sophisticated and state-of-theart multigrid methods designed to take advantage of the structure of the discrete linearized Navier-Stokes equations. In addition, there is a choice of iterative strategies, Picard iteration or Newton's method, for solving the nonlinear algebraic systems arising from the latter problem.

A posteriori error analysis in problems related to fluid dynamics is a subject that has received a lot of attention during the last decades. In the conforming case there are several ways to define error estimators by using the residual equation. in particular, for the Stokes problem, M. Ainsworth, J. Oden [10], C.Carstensen, S.A. Funken [12], D.Kay, D.Silvester [13] and R.Verfurth [14], introduced several error estimators and provided that that they are equivalent to the energy norm of the errors. Other works for the stationary Navier-Stokes problem have been introduced in $[5,8,15,16]$.

The plan of the paper is as follows. Section II presents the model problem used in this paper. The weak formulation is presented in section III. In section IV, we show the existence and uniqueness of the solution.

The discretization by mixed finite elements is described in section V. Section VI introduced two types of a posteriori error bounds of the computed solution. Numerical experiments carried out within the framework of this publication and their comparisons with other results are shown in Section VII.

\section{GOVERNING EQUATIONS}

We will consider the model of viscous incompressible flow in an idealized, bounded, connected domain in $I R^{2}$.

$$
\begin{array}{r}
-v \nabla^{2} \vec{u}+\vec{u} . \nabla \vec{u}+\nabla p=\vec{f} \text { in } \Omega, \\
\nabla \cdot \vec{u}=0 \text { in } \Omega, \\
\vec{n}^{T}(p I-v \nabla \vec{u})=\vec{u}^{T} A-\vec{g} \text { on } \Gamma .
\end{array}
$$

We also assume that $\Omega$ has a polygonal boundary $\Gamma:=\partial \Omega$, so $\vec{n}$ that is the usual outward-pointing normal. 
The vector field $\vec{u}$ is the velocity of the flow and the scalar variable $p$ represents the pressure.

Our mathematical model is the Navier-stoks system with a new boundary condition (3) noted $C_{a, b, c}$. where $v \succ 0$ a given constant is called the kinematic viscosity, $\nabla$ is the gradient, $\nabla$. is the divergence and $\nabla^{2}$ is the Laplacien operator, $\vec{f} \in L^{2}(\Omega), \quad \vec{g} \in L^{2}(\Gamma)$ and $A$ is a real matrix defined as

- $A(x, y)=\left[\begin{array}{ll}a(x, y) & c(x, y) \\ c(x, y) & b(x, y)\end{array}\right]$ for all $(x, y) \in \Gamma$

- There are two strictly positive constants $\alpha_{1}$ and $\beta_{1}$, such that:

$$
\alpha_{1} \leq X^{T} A(x, y) X \leq \beta_{1}
$$

for all $(x, y) \in \Gamma$ and

$X \in S=\left\{X \in I R^{2} /\|X\|_{2}=1\right\}$. Where $a, b$ and $c$ are the function continuous defined on $\Gamma$.

\section{The WeAK Formulation}

We define the following spaces:

$$
\begin{aligned}
& h^{1}(\Omega)=\left\{u: \Omega \rightarrow I R / u ; \frac{\partial u}{\partial x} ; \frac{\partial u}{\partial y} \in L^{2}(\Omega)\right\} \\
& H^{1}(\Omega)=\left[h^{1}(\Omega)\right]^{2} \\
& L_{0}^{2}(\Omega)=\left\{q \in L^{2}(\Omega) / \int_{\Omega} q=0\right\}, \\
& H_{n, 0}^{1}(\Omega)=\left\{\vec{v} \in H^{1}(\Omega) / \vec{v} \cdot \vec{n}=0 \text { in } \Gamma\right\}, \\
& V_{n, 0}^{1}(\Omega)=\left\{\vec{v} \in H_{n, 0}^{1}(\Omega) / \nabla \cdot \vec{v} .=0 \text { in } \Omega\right\} .
\end{aligned}
$$

The standard weak formulation of the Navier-Stokes flow problem (1) - (2)-(3) is the following:

$$
\begin{gathered}
\text { Find } \vec{u} \in H^{1}(\Omega) \text { and } p \in L^{2}(\Omega) \text { such that } \\
\left\{\begin{array}{r}
\bullet \int_{\Omega} v \nabla \vec{u}: \nabla \vec{v}+\int_{\Omega}(\vec{u} \nabla \vec{u}) \vec{v}+\int_{\Gamma} \vec{u}^{T} A \vec{v}-\int_{\Omega} p \nabla \cdot \vec{v} \\
=\int_{\Omega} \vec{f} \vec{v}+\int_{\Gamma} \vec{g} \vec{v}, \\
\bullet-\int_{\Omega} q \nabla \cdot \vec{u} d s=0,
\end{array}\right.
\end{gathered}
$$

for all $(\vec{v}, q) \in H_{n, 0}^{1}(\Omega) \times L_{0}^{2}(\Omega)$.

Let the bilinear forms

$$
\begin{gathered}
A: H_{n, 0}^{1}(\Omega) \times H_{n, 0}^{1}(\Omega) \rightarrow I R ; \quad B: H_{n, 0}^{1}(\Omega) \times L_{0}^{2}(\Omega) \rightarrow I R \\
d: L_{0}^{2}(\Omega) \times L_{0}^{2}(\Omega) \rightarrow I R . \\
A(\vec{u}, \vec{v})=v \int_{\Omega} \nabla \vec{u}: \nabla \vec{v}+\int_{\Gamma} \vec{u}^{T} A \vec{v} \\
B(\vec{u}, q)=-\int_{\Omega} q \nabla \cdot \vec{u} \\
d(p, q)=\int_{\Omega} p \cdot q
\end{gathered}
$$

And the tri-linear forms

$$
\begin{gathered}
C: H_{n, 0}^{1} \times H_{n, 0}^{1} \times H_{n, 0}^{1} \rightarrow I R ; D: H_{n, 0}^{1} \times H_{n, 0}^{1} \times H_{n, 0}^{1} \rightarrow I R \\
C(\vec{u}, \vec{v}, \vec{z})=\int_{\Omega}(\vec{u} \nabla \vec{v}) \vec{z} \\
D(\vec{u}, \vec{v}, \vec{z})=A(\vec{u}, \vec{v})+C(\vec{u}, \vec{v}, \vec{z})
\end{gathered}
$$

Given the functional $\mathrm{L}: L_{0}^{2}(\Omega) \rightarrow I R$

$$
L(\vec{v})=\int_{\Gamma} \vec{g} \cdot \vec{v}+\int_{\Omega} \vec{f} \cdot \vec{v}
$$

The underlying weak formulation (11) may be restated as: find $(\vec{u}, p) \in H_{n, 0}^{1}(\Omega) \times L_{0}^{2}(\Omega)$ such that

$$
\left\{\begin{array}{l}
A(\vec{u}, \vec{v})+C(\vec{u}, \vec{u}, \vec{v})+B(\vec{v}, q)=L(\vec{v}) \\
B(\vec{u}, q)=0
\end{array}\right.
$$

for all $(\vec{v}, q) \in H_{n, 0}^{1}(\Omega) \times L_{0}^{2}(\Omega)$.

In the sequel we can assume that $\vec{g}=\overrightarrow{0}$.

\section{THE EXISTENCE AND UNIQUENESS OF THE SOLUTION}

In this section we will study the existence and uniqueness of the solution of problem (18), for that we need the following results.

Theorem 4.1. There are two strictly positive constants $c_{1}$ and $c_{2}$ such that:

$$
\mathrm{c}_{1}\|\vec{v}\|_{1, \Omega} \leq\|\vec{v}\|_{J, \Omega} \leq c_{2}\|\vec{v}\|_{1, \Omega} \text { for all } \vec{v} \in H_{n, 0}^{1}(\Omega)
$$

with

$$
\begin{gathered}
\|\vec{v}\|_{J, \Omega}=\left(v \int_{\Omega} \nabla \vec{v}: \nabla \vec{v}+\int_{\Gamma} \vec{v}^{T} A \vec{v}\right)^{\frac{1}{2}} \\
\|\vec{v}\|_{1, \Omega}=\left(|\vec{v}|_{1, \Omega}^{2}+\|\vec{v}\|_{0, \Omega}^{2}\right)^{\frac{1}{2}}
\end{gathered}
$$

Proof. 1) The mapping $\gamma_{0}: H^{1}(\Omega) \rightarrow L^{2}(\Gamma)$ is continuous

(See [6] theorem 1,2), then there exists $c \succ 0$ such that: $\|\vec{v}\|_{0, \Gamma} \leq c\|\vec{v}\|_{1, \Omega}$ for all $\vec{v} \in H^{1}(\Omega)$. Using (5) gives,

$$
\alpha_{1}\|\vec{v}\|_{0, \Gamma}^{2} \leq \int_{\Gamma} \vec{v}^{T} A \vec{v} \leq \beta_{1}\|\vec{v}\|_{O, \Gamma}^{2},
$$

then $\|\vec{v}\|_{J, \Omega} \leq c_{2}\|\vec{v}\|_{1, \Omega}$ for all $\vec{v} \in H^{1}(\Omega)$,

with $c_{2}=\left(\beta_{1} c^{2}+v\right)^{\frac{1}{2}}$.

On the other hand. According to 5.55 in [1], there exists a constant $\rho \succ 0$ such that $\|\vec{v}\|_{0, \Omega}^{2} \leq \rho\left(\|\nabla \vec{v}\|_{0, \Omega}^{2}+\|\vec{v}\|_{0, \Gamma}^{2}\right)$.

Using (22), gives

$$
\begin{array}{r}
\mathrm{c}_{1}\|\vec{v}\|_{1, \Omega} \leq\|\vec{v}\|_{J, \Omega} \text { for all } \vec{v} \in H^{1}(\Omega), \\
\text { with } c_{1}=\left(\frac{\rho C}{v \alpha_{1}}+\frac{1}{v}\right)^{-\frac{1}{2}} \text { and } C=\max \left\{\alpha_{1} ; v\right\} \text {. }
\end{array}
$$

Finally, $\mathrm{c}_{1}\|\vec{v}\|_{1, \Omega} \leq\|\vec{v}\|_{J, \Omega} \leq c_{2}\|\vec{v}\|_{1, \Omega}$ for all $\vec{v} \in H^{1}(\Omega)$. 
This result allows us to prove that $\left(H_{n, 0}^{1}(\Omega),\|\cdot\|_{J, \Omega}\right)$ is a Hilbert space which is obliged condition for to obtain the existence and uniqueness of the solution.

Theorem 4.2. $\left(H_{n, 0}^{1}(\Omega),\|\cdot\|_{J, \Omega}\right)$ is a real Hilbert space.

Proof. $\left(H^{1}(\Omega),\|\|_{1, \Omega}\right)$ is a real space and $H_{n, 0}^{1}(\Omega)$ is closed in $H^{1}(\Omega)$ and $\|\cdot\|_{1, \Omega}$ and $\|\cdot\|_{J, \Omega}$ are equivalent norms, then $\left(H_{n, 0}^{1}(\Omega),\|\|_{J, \Omega}\right)$ is a real Hilbert space for two norms.

\section{Theorem 4.3}

1)

$$
A(\vec{u}, \vec{v}) \leq\|\vec{u}\|_{J, \Omega}\|\vec{v}\|_{J, \Omega}
$$

for all $(\vec{u}, \vec{v}) \in H_{n, 0}^{1}(\Omega) \times H_{n, 0}^{1}(\Omega)$

2) $A$ is $H_{n, 0}^{1}(\Omega)$-elliptic for the norm $\|\cdot\|_{J, \Omega}$ and

$$
A(\vec{v}, \vec{v})=\|\vec{v}\|_{\mathrm{J}, \Omega}^{2}
$$

for all $\vec{v} \in H_{n, 0}^{1}(\Omega)$.

Proof: it is easy.

\section{Theorem 4.4}

1)

$$
B(\vec{v}, q) \leq \sqrt{\frac{2}{v}}\|q\|_{0, \Omega}\|\vec{v}\|_{J, \Omega},
$$

for all $(\vec{v}, q) \in H_{n, 0}^{1}(\Omega) \times L_{0}^{2}(\Omega)$

2) The bilinear form $b$ is satisfies the inf-sup: There exists a constant $\beta \succ \mathbf{O}$ such that

$$
\sup _{\vec{v} \in H_{n, 0}^{1}(\Omega)} \frac{B(\vec{v}, q)}{\|\vec{v}\|_{J, \Omega}} \geq \beta\|q\|_{O, \Omega} \text { for all } q \in L_{0}^{2}(\Omega)
$$

Proof.

1) Let $(\vec{v}, q) \in H_{n, 0}^{1}(\Omega) \times L_{0}^{2}(\Omega)$, we have

$$
\begin{aligned}
B(\vec{v}, q) & \leq\|q\|_{0, \Omega}\|\nabla \cdot \vec{v}\|_{0, \Omega} \\
& \leq \sqrt{2}\|q\|_{0, \Omega}\|\nabla \vec{v}\|_{0, \Omega} \\
& \leq \sqrt{\frac{2}{v}}\|q\|_{0, \Omega}\|\vec{v}\|_{J, \Omega} .
\end{aligned}
$$

2) Let $q \in L_{0}^{2}(\Omega)$, we have

$$
\sup _{\vec{v} \in H_{0}^{1}(\Omega)} \frac{B(\vec{v}, q)}{|\vec{v}|_{1, \Omega}} \geq \beta^{\prime}\|q\|_{0, \Omega}(\text { see }[6]),
$$

since

$$
\begin{gathered}
H_{0}^{1}(\Omega)=\left\{\vec{v} \in H^{1}(\Omega) / \vec{v}=\overrightarrow{\mathrm{O}} \text { in } \Gamma\right\} \subset H_{n, 0}^{1}(\Omega) \\
\text { and }|\vec{v}|_{1, \Omega}=\|\vec{v}\|_{1, \Omega} \text { for all } \vec{v} \in H_{0}^{1}(\Omega) \\
\sup _{\vec{v} \in H_{n, 0}^{1}(\Omega)} \frac{B(\vec{v}, q)}{\|\vec{v}\|_{1, \Omega}} \geq \sup _{\vec{v} \in H_{0}^{1}(\Omega)} \frac{B(\vec{v}, q)}{\|\vec{v}\|_{1, \Omega}} \\
=\sup _{\vec{v} \in H_{0}^{1}(\Omega)} \frac{B(\vec{v}, q)}{|\vec{v}|_{1, \Omega}} \\
\geq \beta^{\prime}\|q\|_{O, \Omega} .
\end{gathered}
$$

Using (19) gives

$$
\sup _{\vec{v} \in H_{n, 0}^{1}(\Omega)} \frac{B(\vec{v}, q)}{\|\vec{v}\|_{J, \Omega}} \geq \beta\|q\|_{O, \Omega}, \text { with } \beta=\frac{\beta^{\prime}}{\mathrm{c}_{2}} .
$$

\section{Theorem 4.5}

1) There exists a constant $m \succ 0$ such that

$$
C(\vec{u}, \vec{v}, \vec{z}) \leq m\|\vec{u}\|_{J, \Omega}\|\vec{v}\|_{J, \Omega}\|\vec{z}\|_{J, \Omega}
$$

for all $(\vec{u}, \vec{v}, \vec{z}) \in H_{n, 0}^{1} \times H_{n, 0}^{1} \times H_{n, 0}^{1}$.

2) $C(\vec{u}, \vec{v}, \vec{z})=-C(\vec{u}, \vec{z}, \vec{v})$

for all $(\vec{u}, \vec{v}, \vec{z}) \in V_{n, 0}^{1} \times V_{n, 0}^{1} \times V_{n, 0}^{1}$.

3) $\quad C(\vec{v}, \vec{u}, \vec{u})=0$

for all $(\vec{u}, \vec{v}) \in V_{n, 0}^{1} \times V_{n, 0}^{1}$.

4) $\quad D(\vec{w}, \vec{v}, \vec{v})=A(\vec{v}, \vec{v})=\|\vec{v}\|_{\mathrm{J}, \Omega}^{2}$

5) $\vec{u}_{m} \rightarrow \vec{u}$ weakly in $V_{n, 0}(\Omega)$ (as $m \rightarrow \infty$ ) imply

that $D\left(\vec{u}_{m}, \vec{u}_{m}, \vec{v}\right) \rightarrow D(\vec{u}, \vec{u}, \vec{v})$

Proof

1) Let $\vec{z}, \vec{u}, \vec{v} \in H_{n, 0}^{1}(\Omega)$, we have

$$
\begin{aligned}
C(\vec{u}, \vec{v}, \vec{z}) & \leq m^{\prime}\|\vec{u}\|_{1, \Omega}|\vec{v}|_{1, \Omega}\|\vec{z}\|_{1, \Omega}(\text { see in }[6]) \\
& \leq \frac{m^{\prime}}{c_{1}^{3}}\|\vec{u}\|_{J, \Omega}\|\vec{v}\|_{J, \Omega}\|\vec{z}\|_{J, \Omega}
\end{aligned}
$$

2) Let $\vec{z}, \vec{u}, \vec{v} \in V_{n, 0}^{1}(\Omega)$, we have

$$
\begin{aligned}
C(\vec{z}, \vec{u}, \vec{v})+C(\vec{z}, \vec{v}, \vec{u}) & =\int_{\Omega} \vec{z} \cdot(\nabla \vec{u} \cdot \vec{v}+\nabla \vec{v} \cdot \vec{u}) \\
& =\int_{\Omega} \vec{z} \cdot \nabla(\vec{u} \cdot \vec{v})
\end{aligned}
$$

By Green formula, we have

$$
C(\vec{z}, \vec{u}, \vec{v})+C(\vec{z}, \vec{v}, \vec{u})=\int_{\partial \Omega}(\vec{z} \cdot \vec{n})(\vec{u} \cdot \vec{v})-\int_{\Omega} \operatorname{div} \vec{z} \cdot(\vec{u} \cdot \vec{v})
$$

Since $\vec{z} \in V_{n, 0}(\Omega)$ then $\vec{z} \cdot \vec{n}=0$ and $\operatorname{div} \vec{z}=0$,

finally $C(\vec{z}, \vec{u}, \vec{v})=-C(\vec{z}, \vec{v}, \vec{u})$.

3) It's easy, just take $\vec{v}=\vec{u}$ in (28).

4) It' suffices to apply (29).

5) The same proof of V.Girault and P.A. Raviart in [6] page 115.

According the theorems 1.2 and 1.4, chapter IV in [6], the results (18)-(30) ensure the existence at least one pair $(\vec{u}, p) \in H_{n, 0}^{1}(\Omega) \times L_{0}^{2}(\Omega)$ satisfies (18).

We define

$$
\begin{array}{r}
N=\sup _{\vec{u}, \vec{v}, \vec{z} \in H_{n, 0}^{1}} \frac{C(\vec{u}, \vec{v}, \vec{z})}{\|\vec{u}\|_{J, \Omega}\|\vec{v}\|_{J, \Omega}\|\vec{z}\|_{J, \Omega}} \\
\|\vec{f}\|_{*}=\sup _{\vec{v} \in V_{n, 0}} \frac{\int_{\Omega} \vec{f} \cdot \vec{v}}{\|\vec{v}\|_{J, \Omega}}
\end{array}
$$

Then a well-know (sufficient) condition for uniqueness is that forcing function is small in the sense that $\|\vec{f}\|_{*} \leq \frac{1}{N}$ (it suffices to apply theorems 1.3 and 1.4 chapter IV in [6]). 
Theorem 4.6. Assume that $v$ and $\vec{f} \in L^{2}(\Omega)$ satisfy the following condition

$$
\left|\int_{\Omega} \vec{f} \cdot \vec{v}\right| \leq \frac{\delta}{N}\|\vec{v}\|_{J, \Omega} \text { for all } \vec{v} \in H_{n, 0}^{1}(\Omega)
$$

For some fixed number $\delta \in[0,1[$.

Then there exists an unique $(\vec{u}, p) \in H_{n, 0}^{1}(\Omega) \times L_{0}^{2}(\Omega)$ satisfies (18), and holds

$$
\|\vec{u}\|_{J, \Omega} \leq \frac{\delta}{N}
$$

Proof. The some proof of theorem 2.4 chapter IV in [6].

\section{MiXed Finite ElEMENT APPROXIMATION}

In this section we assume that $\vec{f}, a, b$ and $c$ are the polynomials.

Let $T_{h} ; h \succ 0$, be a family of rectangulations of $\Omega$. For any $T \in T_{h}, \omega_{T}$ is of rectangles sharing at least one edge with element $\mathrm{T}, \widetilde{\omega}_{T}$ is the set of rectangles sharing at least one vertex with T. Also, for an element edge E, $\omega_{E}$ denotes the union of rectangles sharing E, while $\widetilde{\omega}_{E}$ is the set of rectangles sharing at least one vertex whit $\mathrm{E}$.

Next, $\partial \boldsymbol{T}$ is the set of the four edges of $\mathrm{T}$ we denote by $\varepsilon(T)$ and $N_{T}$ the set of its edges and vertices, respectively.

We let $\varepsilon_{h}=\cup_{T \in T_{h}} \varepsilon(T)$ denotes the set of all edges split into interior and boundary edges.

$$
\begin{gathered}
\varepsilon_{h}=\varepsilon_{h, \Omega} \cup \varepsilon_{h, \Gamma} \\
\text { Where } \varepsilon_{h, \Omega}=\left\{E \in \varepsilon_{h}: E \subset \Omega\right\} \\
\varepsilon_{h, \Gamma}=\left\{E \in \varepsilon_{h}: E \subset \partial \Omega\right\}
\end{gathered}
$$

We denote by $h_{T}$ the diameter of a simplex, by $h_{T}$ the diameter of a face $\mathrm{E}$ of T, and we set $h=\max _{T \in T_{h}}\left\{h_{T}\right\}$.

A discrete weak formulation is defined using finite

dimensional spaces $\mathrm{X}_{\mathrm{h}}^{1} \subset H_{n, 0}^{1}(\Omega)$ and $\mathrm{M}^{\mathrm{h}} \subset L_{0}^{2}(\Omega)$

The discrete version of (15) is:

$$
\left\{\begin{array}{l}
\text { find } \vec{u}_{h} \in X_{h}^{1} \text { and } p_{h} \in M^{h} \text { such that: } \\
A\left(\vec{u}_{h}, \vec{v}_{h}\right)+C\left(\vec{u}_{h}, \vec{u}_{h}, \vec{v}_{h}\right)+B\left(\vec{v}_{h}, p_{h}\right)=L\left(\vec{v}_{h}\right) \\
B\left(\vec{v}_{h}, p_{h}\right)=0
\end{array}\right.
$$

For all $\vec{u}_{h} \in X_{h}^{1}$ and $q_{h} \in M^{h}$.

We define the appropriate bases for the finite element spaces, leading to non linear system of algebraic equations. Linearization of this system using Newton iteration gives the finite dimensional

System:

find $\delta \vec{u}_{h} \in X_{h}^{1}$ and $\delta p_{h} \in M^{h}$ such that :

$$
\left\{\begin{array}{c}
v \int_{\Omega} \nabla \delta \vec{u}_{h}: \nabla \vec{v}_{h}+\int_{\alpha \Omega} \delta \vec{u}_{h}^{T} A \vec{v}_{h}+C\left(\delta \vec{u}_{h}, \vec{u}_{h}, \vec{v}_{h}\right) \\
+C\left(\vec{u}_{h}, \delta \vec{u}_{h}, \vec{v}_{h}\right)+B\left(\vec{v}_{h}, \delta p_{h}\right)=R_{k}\left(\vec{v}_{h}\right) \\
B\left(\delta \vec{u}_{h}, q_{h}\right)=r_{k}\left(q_{h}\right)
\end{array}\right.
$$

For all $\vec{u}_{h} \in X_{h}^{1}$ and $q_{h} \in M^{h}$.

Here, $R_{k}\left(\vec{v}_{h}\right)$ and $r_{k}\left(q_{h}\right)$ are the non linear residuals associated with the discrete formulations (36). To define the corresponding linear algebra problem, we use a set of vectorvalued basis functions $\left\{\vec{\varphi}_{i}\right\}_{i=1, \ldots, n_{u}}$ So that

$$
\vec{u}_{h}=\sum_{j=1}^{n_{u}} u_{j} \vec{\varphi}_{j} ; \delta \vec{u}_{h}=\sum_{j=1}^{n_{u}} \Delta u_{j} \vec{\varphi}_{j} .
$$

We introduce a set of pressure basis functions $\left\{\psi_{k}\right\}_{k=1, \ldots, n_{p}}$ and set

$$
p_{h}=\sum_{k=1}^{n_{p}} p_{k} \psi_{k} ; \delta p_{h}=\sum_{k=1}^{n_{p}} \Delta p_{k} \psi_{k} .
$$

Where $n_{u}$ and $n_{p}$ are the numbers of velocity and pressure basis functions, respectively.

We find that the discrete formulation (37) can be expressed as a system of linear equations

$$
\left(\begin{array}{cc}
A_{0}+N+W & B_{0}^{T} \\
B_{0} & 0
\end{array}\right)\left(\begin{array}{l}
\Delta U \\
\Delta P
\end{array}\right)=\left(\begin{array}{l}
f \\
0
\end{array}\right) .
$$

The system is referred to as the discrete Newton problem.

The matrix $A_{0}$ is the vector Laplacian matrix and $B_{0}$ is the divergence matrix

$$
\begin{gathered}
A_{0}=\left[a_{i, j}\right] ; a_{i, j}=v \int_{\Omega} \nabla \vec{\varphi}_{i}: \nabla \vec{\varphi}_{j}+\int_{\Gamma} \vec{\varphi}_{i}^{T} A \vec{\varphi} \\
B_{0}=\left[b_{k, j}\right] ; b_{k, j}=-\int_{\Omega} \psi_{k} \nabla \cdot \vec{\varphi}_{j}
\end{gathered}
$$

The vector-convection matrix $\mathrm{N}$ and the Newton derivative matrix $\mathrm{W}$ are given by

$$
\begin{gathered}
N=\left[n_{i, j}\right] ; n_{i, j}=\int_{\Omega}\left(\vec{u}_{h} \cdot \nabla \vec{\varphi}_{i}\right) \vec{\varphi}_{j} \\
W=\left[w_{i, j}\right] ; w_{i, j}=\int_{\Omega}\left(\vec{\varphi}_{i} \cdot \nabla \vec{u}_{h}\right) \vec{\varphi}_{j}
\end{gathered}
$$

For $i ; j=1, \ldots, n_{u}$ and $k=1, \ldots, n_{p}$.

The right-hand side vectors in (40) are

$$
f=\left[f_{i}\right] ; f_{i}=\int_{\Omega} \vec{f} \cdot \vec{\varphi}_{i}+\int_{\Gamma} \vec{g} \vec{\varphi}_{i},
$$

for $i=1, \ldots, n_{u}$,

For Picard iteration, we give the discrete problem

$$
\left(\begin{array}{cc}
A_{0}+N & B_{0}^{T} \\
B_{0} & 0
\end{array}\right)\left(\begin{array}{l}
\Delta U \\
\Delta P
\end{array}\right)=\left(\begin{array}{l}
f \\
0
\end{array}\right) .
$$

\section{A POSTERIORI ERROR ESTIMATOR}

In this section we propose two types of a posteriori error indicator, a residual error estimator and local Poisson problem 
estimator, which are shown to give global error estimates that are equivalent to the true error.

\section{A. A Residual Error Estimator}

The bubble functions on the reference element $\widetilde{T}=(0,1) \times(0,1)$ are defined as follows:

$$
\begin{gathered}
b_{\widetilde{T}}=2^{4} x(1-x) y(1-y) \\
b_{\widetilde{E}_{1}, \widetilde{T}}=2^{2} x(1-x)(1-y) \\
b_{\widetilde{E}_{2}, \widetilde{T}}=2^{2} y(1-y) x \\
b_{\widetilde{E}_{3}, \widetilde{T}}=2^{2} y(1-x) x \\
b_{\widetilde{E}_{4}, \widetilde{T}}=2^{2} y(1-y)(1-x)
\end{gathered}
$$

Here $b_{\widetilde{T}}$ is the reference element bubble function, and $b_{\widetilde{E}_{i}, \widetilde{T}}, i=1: 4$ are reference edge bubble functions. For any $T \in T_{h}$, the element bubble functions is $b_{T}=b_{\widetilde{T}} \circ F_{T}$ and the element edge bubble function is $b_{E_{i}, T}=b_{\widetilde{E}_{i}, \widetilde{T}} \circ F_{T}$, where $F_{T}$ the affine map form $\tilde{T}$ to $\mathrm{T}$.

For an interior edge $E \in \varepsilon_{h, \Omega}, b_{E}$ is defined piecewise, so that $b_{E / T_{i}}=b_{E, T_{i}}, i=1: 2$ where $\quad \mathrm{E}=\overline{\mathrm{T}}_{1} \cap \overline{\mathrm{T}}_{2}$.

For a boundary edge $\mathrm{E} \in \varepsilon_{h, \Gamma}, b_{E}=b_{E, T}$, where $\mathrm{T}$ is the rectangle such that $\mathrm{E} \in \partial \boldsymbol{T}$.

With these bubble functions, ceruse et al ([3], lemma 4.1] established the following lemma.

Lemma 6.1. Let $\mathrm{T}$ be an arbitrary rectangle in $T_{h}$ and For any $\vec{v}_{T} \in P_{k_{0}}(T)$ and $\vec{v}_{E} \in P_{k_{0}}(E)$, the following inequalities hold.

$$
\begin{aligned}
& c_{k}\left\|\vec{v}_{T}\right\|_{\mathrm{O}, T} \leq\left\|\vec{v}_{T} b_{T}^{\frac{1}{2}}\right\|_{\mathrm{O}, T} \leq C_{k}\left\|\vec{v}_{T}\right\|_{\mathrm{O}, T} \\
& \left|\vec{v}_{T} b_{T}\right|_{1, T} \leq C_{k} h_{T}^{-1}\left\|\vec{v}_{T}\right\|_{0, T} \\
& c_{k}\left\|\vec{v}_{E}\right\|_{\mathrm{O}, E} \leq\left\|\vec{v}_{E} b_{E}^{\frac{1}{2}}\right\|_{0, E} \leq C_{k}\left\|\vec{v}_{E}\right\|_{\mathrm{O}, E} \\
& \left\|\vec{v}_{E} b_{E}\right\|_{0, T} \leq C_{k} h_{E}^{\frac{1}{2}}\left\|\vec{v}_{E}\right\|_{0, E} \\
& \left|\vec{v}_{E} b_{E}\right|_{1, T} \leq C_{k} h_{E}^{-\frac{1}{2}}\left\|\vec{v}_{E}\right\|_{0, E}
\end{aligned}
$$

Where $c_{k}$ and $C_{k}$ are tow constants which only depend on the element aspect ratio and the polynomial degrees $k_{0}$ and $k_{1}$.

Here, $k_{0}$ and $k_{1}$ are fixed and $c_{k}$ and $C_{k}$ can be associated with generic constants $\mathrm{c}$ and $C$ In addition, $\vec{v}_{E}$ which is only defined on the edge $\mathrm{E}$ also denotes its natural extension to the element $\mathrm{T}$.
From the inequalities (50) and (51), we established the following lemma:

Lemma 6.2. Let $\mathrm{T}$ be a rectangle and $E \in \partial T \cap \varepsilon_{h, \Gamma}$. For any $\vec{v}_{E} \in P_{k_{0}}(E)$, the following inequalities hold.

$$
\left\|\vec{v}_{E} b_{E}\right\|_{J, T} \leq C_{k} h_{E}^{-\frac{1}{2}}\left\|\vec{v}_{E}\right\|_{0, E}
$$

Proof. Since $\vec{v}_{E} b_{E}=\overrightarrow{0}$ in the other three edges of rectangle

$\mathrm{T}$, it can be extended to the whole of $\Omega$ by setting $\vec{v}_{E} b_{E}=\overrightarrow{0}$ in $\Omega-\bar{T}$, then

$\left\|\vec{v}_{E} b_{E}\right\|_{1, T}=\left\|\vec{v}_{E} b_{E}\right\|_{1, \Omega}$ and $\left\|\vec{v}_{E} b_{E}\right\|_{J, T}=\left\|\vec{v}_{E} b_{E}\right\|_{J, \Omega}$ Using the inequalities (19), (50) and (51) gives

$$
\begin{aligned}
\left\|\vec{v}_{E} b_{E}\right\|_{J, T} & =\left\|\vec{v}_{E} b_{E}\right\|_{J, \Omega} \\
& \leq c_{2}\left\|\vec{v}_{E} b_{E}\right\|_{1, \Omega} \\
& =c_{2}\left\|\vec{v}_{E} b_{E}\right\|_{1, T} \\
& =c_{2}\left(\left\|\vec{v}_{E} b_{E}\right\|_{0, T}^{2}+\left|\vec{v}_{E} b_{E}\right|_{1, T}^{2}\right)^{\frac{1}{2}} \\
& \leq c_{2} C_{k}\left(h_{E}+h_{E}^{-1}\right)^{\frac{1}{2}}\left\|\vec{v}_{E}\right\|_{0, E} \\
& \leq c_{2} C_{k}\left(D^{2}+1\right)^{\frac{1}{2}} h_{E}^{-\frac{1}{2}}\left\|\vec{v}_{E}\right\|_{0, E} \\
& \leq C h_{E}^{-\frac{1}{2}}\left\|\vec{v}_{E}\right\|_{O, E}
\end{aligned}
$$

With $\mathrm{D}$ is the diameter of $\Omega$ and $C=c_{2} C_{k}\left(D^{2}+1\right)^{\frac{1}{2}}$.

We recall some quasi-interpolation estimates in the following lemma.

Lemma 6.3. Clement interpolation estimate: Given $\overrightarrow{\mathrm{v}} \in H^{1}(\Omega)$, let $\vec{v}_{h} \in \mathrm{X}_{\mathrm{h}}^{1}$ be the quasi-interpolant of $\vec{v}$ defined by averaging as in [4]. For any $T \in T_{h}$,

$$
\left\|\vec{v}-\vec{v}_{h}\right\|_{0, T} \leq C h_{T}|\vec{v}|_{1, \widetilde{\omega}_{T}},
$$

and for all $E \in \partial T$,

$$
\left\|\vec{v}-\vec{v}_{h}\right\|_{0, E} \leq C h_{E}^{\frac{1}{2}}|\vec{v}|_{1, \widetilde{\omega}_{E}}
$$

We let $(\vec{u}, p)$ denote the solution of (18) and let denote $\left(\vec{u}_{h}, p_{h}\right)$ the solution of (36) with an approximation on a rectangular subdivision $T_{h}$.

Our aim is to estimate the velocity and the pressure errors $\vec{e}=\vec{u}-\vec{u}_{h} \in H_{n, 0}^{1}(\Omega)$ and $\varepsilon=p-p_{h} \in L_{0}^{2}(\Omega)$.

The element contribution $\eta_{R, T}$, of the residual error estimator $\eta_{R}$ is given by

$$
\eta_{R, T}^{2}=h_{T}^{2}\left\|\vec{R}_{T}\right\|_{0, T}^{2}+\left\|R_{T}\right\|_{0, T}^{2}+\sum_{E \in \partial T} h_{E}\left\|\vec{R}_{E}\right\|_{0, E}^{2}
$$

and the components in (55) are given by

$$
\vec{R}_{T}=\left\{\vec{f}+v \nabla^{2} \vec{u}_{h}-\vec{u}_{h} \nabla \vec{u}_{h}-\nabla p_{h}\right\} / T
$$




$$
\begin{gathered}
R_{T}=\left\{\nabla \cdot \vec{u}_{h}\right\} / T \\
\vec{R}_{E}=\left\{\begin{array}{l}
\frac{1}{2}\left[\left|v \nabla \vec{u}_{h}-p_{h} I\right|\right] ; E \in \varepsilon_{h, \Omega} \\
\vec{g}-\left(\vec{u}_{h}^{T} A+\left(v \nabla \vec{u}_{h}-p_{h} I\right) \vec{n}_{E, T}\right) ; E \in \varepsilon_{h, \Gamma}
\end{array}\right.
\end{gathered}
$$

With the key contribution coming from the stress jump associated with an edge $\mathrm{E}$ adjoining elements $\mathrm{T}$ and $\mathrm{S}$ :

$\left[\nu \nabla \vec{u}_{h}-p_{h} I \mid\right]=\left(\nu \nabla \vec{u}_{h}-p_{h} I\right) / T-\left(\nu \nabla \vec{u}_{h}-p_{h} I\right) / S$

The global residual error estimator is given by:

$$
\eta_{R}=\sqrt{\sum_{T \in T_{h}} \eta_{R, T}^{2}}
$$

Our aim is bound $\left\|\overrightarrow{\boldsymbol{u}}-\overrightarrow{\boldsymbol{u}}_{h}\right\|_{X}$ and $\left\|p-p_{h}\right\|$ with respect to the norm $\|\cdot\|_{J}$ for velocity $\|\overrightarrow{\mathrm{v}}\|_{\mathrm{X}}=\|\overrightarrow{\mathrm{v}}\|_{\mathrm{J}, \Omega}$ and the quotient norm for the pressure $\|p\|_{M}=\|p\|_{0, \Omega}$.

For any $T \in T_{h}$, and $E \in \partial T$, we define the following two functions:

$$
\vec{w}_{T}=\vec{R}_{T} b_{T} ; \vec{w}_{E}=\vec{R}_{E} b_{E},
$$

- $\vec{w}_{T}=\overrightarrow{0}$ on $\partial \mathrm{T}$,

- if $E \in \partial T \cap \varepsilon_{h, \Omega}$ then $\vec{w}_{E}=\overrightarrow{0}$ on $\partial \omega_{E}$.

- if $E \in \partial T \cap \varepsilon_{h, \Gamma}$ then $\vec{w}_{E}=\overrightarrow{0}$ in the other edges of rectangle $\mathrm{T}$.

- $\vec{w}_{E}$ and $\vec{w}_{T}$ can be extended to whole of

$\Omega$ by setting:

$\vec{w}_{T}=\overrightarrow{0}$ in $\Omega-\overline{\mathrm{T}}$

$\vec{w}_{E}=\overrightarrow{0}$ in $\Omega-\overline{\omega_{E}}$ if $E \in \partial T \cap \varepsilon_{h, \Omega}$.

$\vec{w}_{E}=\overrightarrow{0}$ in $\Omega-\mathrm{T}$ if $E \in \partial T \cap \varepsilon_{h, \Gamma}$.

With these two functions we have the following lemmas:

Lemma 6.4. For any $T \in T_{h}$ we have:

$$
\int_{T} \vec{f} \cdot \vec{w}_{T}=\int_{T}(v \nabla \vec{u}-p I): \nabla \vec{w}_{T}+\int_{T}(\vec{u} \cdot \nabla \vec{u}) \cdot \vec{w}_{T}
$$

\section{Proof}

Using (1) gives

$\int_{T} \vec{f} \cdot \vec{w}_{T}=\int_{T}-\left(v \nabla^{2} \vec{u}+\vec{u} \cdot \nabla \vec{u}+\nabla p\right) \vec{w}_{T}$

By applying the Green formula and $\vec{w}_{T}=\overrightarrow{0}$ on $\partial \mathrm{T}$, we obtain

$$
\begin{aligned}
\int_{T} \vec{f} \cdot \vec{w}_{T} & =-\int_{\partial T}(\nu \nabla \vec{u}-p I) \vec{n} \cdot \vec{w}_{T} \\
& +\int_{T}(\nu \nabla \vec{u}-p I): \nabla \vec{w}_{T}+\int_{T}(\vec{u} \cdot \nabla \vec{u}) \cdot \vec{w}_{T} \\
& =\int_{T}(\nu \nabla \vec{u}-p I): \nabla \vec{w}_{T}+\int_{T}(\vec{u} \cdot \nabla \vec{u}) \cdot \vec{w}_{T} .
\end{aligned}
$$

\section{Lemma 6.5}

i) if $E \in \partial T \cap \varepsilon_{h, \Omega}$ we have :

$$
\int_{\omega_{E}} \vec{f} \cdot \vec{w}_{E}=\int_{\omega_{E}}(\nu \nabla \vec{u}-p I): \nabla \vec{w}_{E}+\int_{\omega_{E}}(\vec{u} \cdot \nabla \vec{u}) \cdot \vec{w}_{E}
$$

ii) if $E \in \partial T \cap \varepsilon_{h, \Gamma}$, We have:

$$
\begin{aligned}
\int_{T} \vec{f} \cdot \vec{w}_{E} & =\int_{T}(v \nabla \vec{u}-p I): \nabla \vec{w}_{E} \\
& +\int_{\partial T}\left(\vec{u}^{T} A-\vec{g}\right) \vec{w}_{E}+\int_{T}(\vec{u} \cdot \nabla \vec{u}) \cdot \vec{w}_{T}
\end{aligned}
$$

Proof.

i) The same proof of (56).

ii) If $E \in \partial T \cap \varepsilon_{h, \Gamma}$, We have :

$$
\begin{aligned}
\int_{T} \vec{f} \cdot \vec{w}_{T}=\int_{T}-\left(\nu \nabla^{2} \vec{u}+\vec{u} \cdot \nabla \vec{u}+\nabla p\right) \cdot \vec{w}_{T} \\
=\int_{T}(\nu \nabla \vec{u}-p I): \nabla \vec{w}_{E}-\int_{\partial T}(\nu \nabla \vec{u}-p I) \vec{n} \cdot \vec{w}_{E} \\
\quad+\int_{T}(\vec{u} \cdot \nabla \vec{u}) \cdot \vec{w}_{T}
\end{aligned}
$$

We have

$\vec{n}^{T}(p I-v \nabla \vec{u})=\vec{u}^{T} A-\vec{g} \quad$ on $E \subset \Gamma$ and $\vec{w}_{E}=\overrightarrow{0}$ in the other tree edges of rectangle $\mathrm{T}$, then

$$
\begin{aligned}
\int_{T} \vec{f} \cdot \vec{w}_{E}= & \int_{T}(\nu \nabla \vec{u}-p I): \nabla \vec{w}_{E} \\
& +\int_{\partial T}\left(\vec{u}^{T} A-\vec{g}\right) \vec{w}_{E}+\int_{T}(\vec{u} \cdot \nabla \vec{u}) \cdot \vec{w}_{T} .
\end{aligned}
$$

We define the bilinear form

$$
G((\vec{u}, \vec{p}) ;(\vec{v}, q))=A(\vec{u}, \vec{v})+B(\vec{u}, q)+B(\vec{v}, p)
$$

We define also the following functional

$$
\theta(K, \vec{x}, \vec{y}, \vec{v})=\int_{K}(\vec{x} . \nabla x) \cdot \vec{v}-\int_{K}(\vec{y} . \nabla \vec{y}) \cdot \vec{v}
$$

for all $K \subset \Omega$ and $\vec{x}, \vec{y}, \vec{v} \in H_{n, 0}^{1}(\Omega)$.

Lemma 6.6. There exists $C \succ 0$ and $h_{0} \succ 0$ such that

$$
\theta\left(K, \vec{u}, \vec{u}_{h}, \vec{v}\right) \leq C\|\vec{e}\|_{J, K}\|\vec{v}\|_{J, K},
$$

for all $h \leq h_{0}, \vec{v} \in H_{n, 0}^{1}(\Omega)$ and $K \subset \Omega$.

Proof. Using (27), (32) and (35), we have that for $\vec{v} \in H_{n, 0}^{1}(\Omega)$

$$
\begin{aligned}
\theta(K, \vec{u} & \left., \vec{u}_{h}, \vec{v}\right)=\int_{K}(\vec{u} \cdot \nabla \vec{u}) \cdot \vec{v}-\int_{K}\left(\vec{u}_{h} \cdot \nabla \vec{u}_{h}\right) \cdot \vec{v} \\
& =\int_{K}(\vec{u} \cdot \nabla \vec{e}) \cdot \vec{v}+\int_{K}\left(\vec{e} \cdot \nabla \vec{u}_{h}\right) \cdot \vec{v} \\
& \leq N\left(\|\vec{u}\|_{J, K}\|\vec{e}\|_{J, K}\|\vec{v}\|_{J, K}+\|\vec{e}\|_{J, K}\left\|\vec{u}_{h}\right\|_{J, K} \cdot\|\vec{v}\|_{J, K}\right) \\
& \leq N\left(2\|\vec{u}\|_{J, K}\|\vec{e}\|_{J, K}\|\vec{v}\|_{J, K}+\|\vec{e}\|_{J, K}^{2} \cdot\|\vec{v}\|_{J, K}\right) \\
& \leq\left(2 \delta+N\|\vec{e}\|_{J, K}\right)\|\vec{e}\|_{J, K}\|\vec{v}\|_{J, K}
\end{aligned}
$$

We have

$\lim _{h \rightarrow 0} \vec{u}_{h}=\vec{u}$ in $H_{n, 0}^{1}(\Omega)$, then there exists $h_{0} \succ 0$ such that $\|\vec{e}\|_{J, K}=\left\|\vec{u}-\vec{u}_{h}\right\|_{J, K} \leq 1$ for all $h \leq h_{0}$.

Using this result and (60), we obtain

$$
\theta\left(K, \vec{u}, \vec{u}_{h}, \vec{v}\right) \leq C\|\vec{e}\|_{J, K}\|\vec{v}\|_{J, K}
$$

for all $h \leq h_{0}$ and $\vec{v} \in H_{n, 0}^{1}(\Omega)$, with $\mathrm{C}=2 \delta v+N$. 
We have

$$
\begin{aligned}
G((\vec{e}, \varepsilon) ;(\vec{v}, q))= & A\left(\vec{u}-\vec{u}_{h}, \vec{v}\right)+B\left(\vec{u}-\vec{u}_{h}, q\right)+B\left(\vec{v}, p-p_{h}\right) \\
= & G((\vec{u}, p) ;(\vec{v}, q))-G\left(\left(\vec{u}_{h}, p_{h}\right) ;(\vec{v}, q)\right) \\
= & L(\vec{v})-C(\vec{u}, \vec{u}, \vec{v})-G\left(\left(\vec{u}_{h}, p_{h}\right) ;(\vec{v}, q)\right) \\
= & L(\vec{v})-C(\vec{u}, \vec{u}, \vec{v})-A\left(\vec{u}_{h}, \vec{v}\right)-B\left(\vec{v}, p_{h}\right) \\
& -B\left(\vec{u}_{h}, q\right) .
\end{aligned}
$$

Then

$$
\begin{array}{r}
G((\vec{e}, \varepsilon) ;(\vec{v}, q))+\theta\left(\Omega, \vec{u}, \vec{u}_{h}, \vec{v}\right)=-C\left(\vec{u}_{h}, \vec{u}_{h}, \vec{v}\right)+L(\vec{v}) \\
-A\left(\vec{u}_{h}, \vec{v}\right)-B\left(\vec{v}, p_{h}\right)-B\left(\vec{u}_{h}, q\right)
\end{array}
$$

for $\quad$ all $(\vec{v}, q) \in H_{n, 0}^{1}(\Omega) \times L_{0}^{2}(\Omega)$.

We find that theerrors $\vec{e} \in H_{n, 0}^{1}(\Omega)$ and $\varepsilon \in L_{0}^{2}(\Omega)$

Satisfy the non-linear equation

$$
\begin{aligned}
& G((\vec{e}, \varepsilon) ;(\vec{v}, q))+\theta\left(\Omega, \vec{u}, \vec{u}_{h}, \vec{v}\right) \\
& \quad=\sum_{T \in T_{h}}\left\{\left(\vec{R}_{T}, \vec{v}\right)_{T}-\sum_{E \subset \partial T}\left(\vec{R}_{E}, \vec{v}\right)_{E}+\left(R_{T}, q\right)_{T}\right\}
\end{aligned}
$$

for all $(\vec{v}, q) \in H_{n, 0}^{1}(\Omega) \times L_{0}^{2}(\Omega)$.

We define a pair $(\vec{\varphi}, \psi) \in H_{n, 0}^{1}(\Omega) \times L_{0}^{2}(\Omega)$

to be the Ritz projection of the modified residuals

$$
\begin{aligned}
A(\vec{\varphi}, \vec{v})+d(\psi, q) & =A(\vec{e}, \vec{v})+B(\vec{e}, q)+B(\vec{v}, \varepsilon)+\theta\left(\Omega, \vec{u}, \vec{u}_{h}, \vec{v}\right) \\
& =G((\vec{e}, \varepsilon) ;(\vec{v}, q))+\theta\left(\Omega, \vec{u}, \vec{u}_{h}, \vec{v}\right)
\end{aligned}
$$

for all $(\vec{v}, q) \in H_{n, 0}^{1}(\Omega) \times L_{0}^{2}(\Omega)$.

\section{Lemma 6.7}

$$
A\left(\vec{\varphi}, \vec{v}_{h}\right)=0 \text { for all } \vec{v}_{h} \in X_{h}^{1}
$$

Proof: we set $q=0$ and $\vec{v}=\vec{v}_{h}$ in (62), we obtain

$$
\begin{aligned}
A\left(\vec{\varphi}, \vec{v}_{h}\right) & =A\left(\vec{e}, \vec{v}_{h}\right)+B\left(\vec{v}_{h}, \varepsilon\right)+\theta\left(\Omega, \vec{u}, \vec{u}_{h}, \vec{v}_{h}\right) \\
& =A\left(\vec{u}, \vec{v}_{h}\right)-A\left(\vec{u}_{h}, \vec{v}_{h}\right)+B\left(\vec{v}_{h}, p\right)-B\left(\vec{v}_{h}, p_{h}\right) \\
& +C\left(\vec{u}, \vec{u}, \vec{v}_{h}\right)-C\left(\vec{u}_{h}, \vec{u}_{h}, \vec{v}_{h}\right) \\
& =L\left(\vec{v}_{h}\right)-L\left(\vec{v}_{h}\right)=0 .
\end{aligned}
$$

Next, we establish the equivalence between the norms of $(\vec{e}, \varepsilon) \in H_{n, 0}^{1}(\Omega) \times L_{0}^{2}(\Omega)$ and the norms of the solution $(\vec{\varphi}, \psi) \in H_{n, 0}^{1}(\Omega) \times L_{0}^{2}(\Omega)$ of (63).

Theorem 6.8. Let the conditions of theorem 4.6 hold. There exist two positive constants $k_{1}$ and $k_{2}$, independent of $h$, such that

$k_{1}\left\{\|\vec{\varphi}\|_{J, \Omega}^{2}+\|\psi\|_{0, \Omega}^{2}\right\} \leq\left\{\|\vec{e}\|_{J, \Omega}^{2}+\|\varepsilon\|_{0, \Omega}^{2}\right\} \leq k_{2}\left\{\|\vec{\varphi}\|_{J, \Omega}^{2}+\|\psi\|_{0, \Omega}^{2}\right\}$

Proof. The same proof of theorem 3 in [9].

Theorem 6.9.

For all $(\vec{w}, s) \in H_{n, 0}^{1}(\Omega) \times L_{0}^{2}(\Omega)$, we have

$$
\sup _{(\vec{v}, q) \in H_{n, 0}^{1} \times L_{0}^{2}} \frac{A(\vec{w}, \vec{v})+d(s, q)}{\|\vec{v}\|_{J, \Omega}+\|q\|_{0, \Omega}} \geq \frac{1}{2}\left(\|\vec{w}\|_{J, \Omega}+\|s\|_{0, \Omega}\right) .
$$

Proof. Let $(\vec{w}, s) \in H_{n, 0}^{1}(\Omega) \times L_{0}^{2}(\Omega)$, we have

$$
\begin{aligned}
\sup _{(\vec{v}, q) \in H_{n, 0}^{1} \times L_{0}^{2}} \frac{A(\vec{w}, \vec{v})+d(s, q)}{\|\vec{v}\|_{J, \Omega}+\|q\|_{0, \Omega}} & \geq \frac{A(\vec{w}, \vec{w})+d(s, 0)}{\|\vec{v}\|_{J, \Omega}+\|0\|_{0, \Omega}} \\
= & \frac{A(\vec{w}, \vec{w})}{\|\vec{w}\|_{J, \Omega}}=\|\vec{w}\|_{J, \Omega}
\end{aligned}
$$

We also have

$$
\begin{aligned}
\sup _{(\vec{v}, q) \in H_{n, 0}^{1} \times L_{0}^{2}} \frac{A(\vec{w}, \vec{v})+d(s, q)}{\|\vec{v}\|_{J, \Omega}+\|q\|_{0, \Omega}} & \geq \frac{A(\vec{w}, 0)+d(s, s)}{\|0\|_{J, \Omega}+\|s\|_{0, \Omega}} \\
& =\frac{d(s, s)}{\|s\|_{0, \Omega}}=\|s\|_{0, \Omega} .
\end{aligned}
$$

We gather (66) and (67) to get

$$
\sup _{(\vec{v}, q) \in H_{n, 0}^{1} \times L_{0}^{2}} \frac{A(\vec{w}, \vec{v})+d(s, q)}{\|\vec{v}\|_{J, \Omega}+\|q\|_{0, \Omega}} \geq \frac{1}{2}\left(\|\vec{w}\|_{J, \Omega}+\|s\|_{0, \Omega}\right) .
$$

Theorem 6.10. For any mixed finite element approximation (not necessarily inf-sup stable) defined on rectangular grids $T_{h}$, the residual estimator $\eta_{R}$ satisfies:

$$
\|\vec{e}\|_{J, \Omega}+\|\varepsilon\|_{o, \Omega} \leq C \eta_{R}
$$

and $\eta_{R, T} \leq C\left(\sum_{T^{\prime} \in \omega_{T}}\left\{\|\vec{e}\|_{J, T^{\prime}}^{2}+\|\varepsilon\|_{0, T^{\prime}}^{2}\right\}\right)^{\frac{1}{2}}$.

Note that the constant $\mathrm{C}$ in the local lower bound is independent of the domain, and

$$
\|\vec{e}\|_{J, T}^{2}=v \int_{T} \nabla \vec{e}: \nabla \vec{e}+\int_{\partial T}^{\vec{e}^{T}} A \cdot \vec{e} .
$$

Proof. To establish the upper bound we let $(\vec{v}, q) \in H_{n, 0}^{1}(\Omega) \times L_{0}^{2}(\Omega)$ and $\vec{v}_{h} \in X_{h}^{1}$ be the clement interpolation of $\vec{v}$. Using (63), (61) and (62), give 
$A(\vec{\varphi}, \vec{v})+d(\psi, q)=A\left(\vec{\varphi}, \vec{v}-\vec{v}_{h}\right)+d(\psi, q)$

$$
\begin{aligned}
= & \sum_{T \in T_{h}}\left\{\left(\vec{R}_{T}, \vec{v}-\vec{v}_{h}\right)_{T}-\sum_{E \in \partial T}\left(\vec{R}_{E}, \vec{v}-\vec{v}_{h}\right)_{E}+\left(R_{T}, q\right)_{T}\right\} \\
\leq & \sum_{T \in T_{h}}\left\{\left\|\vec{R}_{T}\right\|_{0, T}\left\|\vec{v}-\vec{v}_{h}\right\|_{0, T}+\sum_{T \in \hat{\sigma} T}\left\|\vec{R}_{E}\right\|_{0, T}\left\|\vec{v}-\vec{v}_{h}\right\|_{0, E}\right. \\
& \left.+\|q\|_{0, T}\left\|R_{T}\right\|_{0, E}\right\} \\
\leq & \left(\sum_{T \in T_{h}} h_{T}^{2}\left\|\vec{R}_{T}\right\|_{0, T}^{2}\right)^{\frac{1}{2}}\left(\sum_{T \in T_{h}} \frac{1}{h_{T}^{2}}\left\|\vec{v}-\vec{v}_{h}\right\|_{0, T}^{2}\right)^{\frac{1}{2}} \\
& +\left(\sum_{T \in T_{h} E \in \partial T} h_{E}\left\|\vec{R}_{E}\right\|_{0, T}^{2}\right)^{\frac{1}{2}}\left(\sum_{T \in T_{h} E \in \partial T} \frac{1}{h_{E}}\left\|\vec{v}-\vec{v}_{h}\right\|_{0, E}^{2}\right)^{\frac{1}{2}}
\end{aligned}
$$

Using (52) and (53), then gives

$$
\begin{aligned}
& A(\vec{\varphi}, \vec{v})+d(\psi, q) \leq C\left(\sum_{T \in T_{h}}\left\{\|\vec{v}\|_{J, T}^{2}+\|q\|_{0, T}^{2}\right\}\right)^{\frac{1}{2}} \\
& \times\left(\sum_{T \in T_{h}}\left\{h_{T}^{2}\left\|\vec{R}_{T}\right\|_{0, T}^{2}+\left\|R_{T}\right\|_{0, T}^{2}+\sum_{E \in O T} h_{E}\left\|\vec{R}_{E}\right\|_{0, E}^{2}\right\}\right)^{\frac{1}{2}} .
\end{aligned}
$$

Finally, using (65) gives:

$$
\|\vec{\varphi}\|_{J, T}+\|\psi\|_{0, T} \leq C \sum_{T \in T_{h}}\left\{h_{T}^{2}\left\|\vec{R}_{T}\right\|_{0, T}^{2}+\left\|R_{T}\right\|_{0, T}^{2}+\sum_{E \in \partial T} h_{E}\left\|\vec{R}_{E}\right\|_{0, E}^{2}\right\}
$$

According to theorem 6.8, we have

$$
\|\vec{e}\|_{J, T}+\|\varepsilon\|_{0, T} \leq C_{\Omega} \sum_{T \in T_{h}}\left\{h_{T}^{2}\left\|\vec{R}_{T}\right\|_{0, T}^{2}+\left\|R_{T}\right\|_{0, T}^{2}+\sum_{E \in \delta T} h_{E}\left\|\vec{R}_{E}\right\|_{0, E}^{2}\right\}
$$

This establishes the upper bound.

Turning to the local lower bound. First, for the element residual part, we have:

$$
\begin{aligned}
\int_{T} \vec{R}_{T} \cdot \vec{w}_{T} & =\int_{T}\left(\vec{f}+v \nabla^{2} \vec{u}_{h}-\vec{u}_{h} \cdot \nabla \vec{u}_{h}-\nabla p_{h}\right) \cdot \vec{w}_{T} \\
& =\int_{T} \vec{f} \cdot \vec{w}_{T}-\int_{T}\left(v \nabla \vec{u}_{h}-p_{h} I\right): \nabla \vec{w}_{T} \\
& +\int_{\partial T}\left(v \nabla \vec{u}_{h}-p_{h} I\right) \vec{n} \cdot \vec{w}_{T}-\int_{T}\left(\vec{u}_{h} \nabla \vec{u}_{h}\right) \cdot \vec{w}_{T}
\end{aligned}
$$

See that $\vec{w}_{T}=\overrightarrow{0}$ in $\partial \mathbf{T}$, using (56) and (57) gives:

$$
\begin{aligned}
\int_{T} \vec{R}_{T} \cdot \vec{w}_{T} & =\int_{T}(v \nabla \vec{e}-\varepsilon I): \nabla \vec{w}_{T}+\theta\left(T, \vec{u}, \vec{u}_{h}, \vec{w}_{T}\right) \\
& \leq \mathrm{C}^{\prime}\left(|\overrightarrow{\mathrm{e}}|_{1, T}+\|\varepsilon\|_{0, T}\right)\left|\vec{w}_{T}\right|_{1, T}+C^{\prime \prime}\|\vec{e}\|_{J, T}\|\vec{w}\|_{J, T}
\end{aligned}
$$

Since $\vec{w}_{T}=\overrightarrow{0}$ in $\partial \mathrm{T}$, then $\|\vec{w}\|_{J, T}=\sqrt{v}\left|\vec{w}_{T}\right|_{1, T}$,

Using (48), gives

$$
\int_{T} \vec{R}_{T} \cdot \vec{w}_{T} \leq \mathrm{C}\left(\|\overrightarrow{\mathbb{e}}\|_{J, T}^{2}+\|\varepsilon\|_{0, T}^{2}\right)^{\frac{1}{2}} h_{T}^{-1}\left\|\vec{R}_{T}\right\|_{0, T}
$$

In addition, from the inverse inequality (47)

$$
\int_{T} \vec{R}_{T} \cdot \vec{w}_{T}=\left\|\vec{R}_{T} b_{T}^{\frac{1}{2}}\right\|_{0, T}^{2} \geq c_{k}\left\|\vec{R}_{T}\right\|_{0, T}^{2},
$$

Thus, $\quad h_{T}^{2}\left\|\vec{R}_{T}\right\|_{0, T}^{2} \leq C\left(\|\overrightarrow{\mathrm{e}}\|_{J, T}^{2}+\|\varepsilon\|_{0, T}^{2}\right)$

Next comes the divergence part,

$$
\begin{aligned}
\left\|R_{T}\right\|_{0, T} & =\left\|\nabla \cdot \vec{u}_{h}\right\|_{0, T} \\
& =\left\|\nabla \cdot\left(\vec{u}-\vec{u}_{h}\right)\right\|_{0, T} \\
& \leq \sqrt{2}\left|\vec{u}-\vec{u}_{h}\right|_{1, T} \\
& \leq \sqrt{\frac{2}{v}}\left\|\vec{u}-\vec{u}_{h}\right\|_{J, T}=\sqrt{\frac{2}{v}}\|\vec{e}\|_{J, T}
\end{aligned}
$$

Finally, we need to estimate the jump term. For an edge if $E \in \partial T \cap \varepsilon_{h, \Omega}$, We have

$$
\begin{aligned}
2 \int_{E} \vec{R}_{E} \cdot \vec{w}_{E}=\sum_{i=1: 2} \int_{\partial T_{i}}\left(\nu \nabla \vec{u}_{h}-p_{h} I\right) \vec{n} \cdot \vec{w}_{E} \\
\quad=\int_{\omega_{E}}\left(\nu \nabla \vec{u}_{h}-p_{h} I\right): \nabla \vec{w}_{E}+\sum_{i=1: 2} \int_{T_{i}}\left(\nu \nabla^{2} \vec{u}_{h}-\nabla p_{h}\right) \cdot \vec{w}_{E} .
\end{aligned}
$$

Using (57) and $\vec{w}_{E}=\overrightarrow{0}$ in $\partial \omega_{\mathrm{E}}$, gives :

$$
\begin{aligned}
& 2 \int_{E} \vec{R}_{E} \cdot \vec{w}_{E}=-\int_{\omega_{E}}(\nu \nabla \vec{e}-\varepsilon I): \nabla \vec{w}_{E} \\
&+\sum_{i=1: 2} \int_{T_{i}} \vec{R}_{T_{i}} \vec{w}_{E}-\theta\left(\omega_{E}, \vec{u}, \vec{u}_{h}, \vec{w}_{E}\right) \\
& \leq\left(v|\vec{e}|_{1, \omega_{E}}+\|\varepsilon\|_{0, \omega_{E}}\right)\left|\vec{w}_{E}\right|_{1, \omega_{E}}+\sum_{i=1: 2}\left\|\vec{R}_{T_{i}}\right\|_{0, T_{i}}\left\|\vec{w}_{E}\right\|_{0, T_{i}} \\
&+C\|\vec{e}\|_{J, \omega_{E}}\left\|\vec{w}_{E}\right\|_{J, \omega_{E}}
\end{aligned}
$$

Since $\vec{w}_{E}=\overrightarrow{0}$ in $\partial \omega_{\mathrm{E}}$, then $\|\vec{w}\|_{J, \omega_{\mathrm{E}}}=\sqrt{v}\left|\vec{w}_{T}\right|_{1, \omega_{\mathrm{E}}}$

Using (50) and (51), to get

$$
\begin{aligned}
2 \int_{E} \vec{R}_{E} \cdot \vec{w}_{E} \leq & C^{\prime}\left(|\vec{e}|_{1, \omega_{E}}^{2}+\|\varepsilon\|_{0, \omega_{E}}^{2}\right)^{\frac{1}{2}} h_{E}^{-\frac{1}{2}}\left\|\vec{R}_{E}\right\|_{0, E} \\
& +\sum_{i=1: 2}\left\|\vec{R}_{T_{i}}\right\|_{0, T_{i}} h_{E}^{\frac{1}{2}}\left\|\vec{R}_{E}\right\|_{0, E}
\end{aligned}
$$

Using (68), gives

$$
2 \int_{E} \vec{R}_{E} \cdot \vec{w}_{E} \leq C\left(\|\vec{e}\|_{J, \omega_{E}}^{2}+\|\varepsilon\|_{0, \omega_{E}}^{2}\right)^{\frac{1}{2}} h_{E}^{-\frac{1}{2}}\left\|\vec{R}_{E}\right\|_{0, E}
$$

Using the inverse inequality (49) and thus using (70) gives

$$
h_{E}\left\|\vec{R}_{E}\right\|_{0, E}^{2} \leq \mathrm{C}\left(\|\overrightarrow{\mathrm{e}}\|_{J, \omega_{E}}^{2}+\|\varepsilon\|_{0, \omega_{E}}^{2}\right)
$$

Also need to show that (70) holds for boundary edges.

For an $E \in \partial T \cap \varepsilon_{h, \Gamma}$, We have

$$
\begin{aligned}
\int_{E} \vec{R}_{E} \cdot \vec{w}_{E} & =\int_{\partial T}\left[\vec{u}_{h}^{T} A+\left(\nu \nabla \vec{u}_{h}-p_{h} I\right) \vec{n}-\vec{g}\right] \vec{w}_{E} \\
& =\int_{\partial T}\left(\vec{u}_{h}^{T} A-\vec{g}\right) \cdot \vec{w}_{E}+\int_{\partial T}\left(\left(\nu \nabla \vec{u}_{h}-p_{h} I\right) \vec{n}\right) \cdot \vec{w}_{E} \\
& =\int_{\partial T}\left(\vec{u}_{h}^{T} A-\vec{g}\right) \cdot \vec{w}_{E}+\int_{T}\left(\left(\nu \nabla \vec{u}_{h}-p_{h} I\right)\right): \nabla \vec{w}_{E} \\
& +\int_{T}\left(\left(\nu \nabla^{2} \vec{u}_{h}-\nabla p_{h}\right)\right) \vec{w}_{E}
\end{aligned}
$$

Using (58), (59) and (4), gives 


$$
\begin{aligned}
\int_{E} \vec{R}_{E} \cdot \vec{w}_{E}= & -\int_{T}((\nu \nabla \vec{e}-\varepsilon I)): \nabla \vec{w}_{E}-\int_{\partial T} \vec{e}^{T} A \cdot \vec{w}_{E} \\
& +\int_{T} \vec{R}_{T} \cdot \vec{w}_{E}-\theta\left(T, \vec{u}, \vec{u}_{h}, \vec{w}_{E}\right) \\
\leq & \left(v|\vec{e}|_{1, T}+\|\varepsilon\|_{0, T}\right)\left|\vec{w}_{E}\right|_{1, T}+\beta_{1}\|\vec{e}\|_{0, \partial T}\left\|\vec{w}_{E}\right\|_{0, \partial T} \\
& +C^{\prime}\|\vec{e}\|_{J, T}\left\|\vec{w}_{E}\right\|_{J, T}+\left\|\vec{R}_{T}\right\|_{0, T}\left\|\vec{w}_{E}\right\|_{0, T} \\
\leq & C\left(\|\vec{e}\|_{J, T}+\|\varepsilon\|_{0, T}\right)\left\|\vec{w}_{E}\right\|_{J, T}+\left\|\vec{R}_{T}\right\|_{0, T}\left\|\vec{w}_{E}\right\|_{0, T}
\end{aligned}
$$

Using (50) and (52) gives

$$
\begin{gathered}
\int_{E} \vec{R}_{E} \cdot \vec{w}_{E} \leq C_{1}\left(\|\vec{e}\|_{J, T}^{2}+\|\varepsilon\|_{O, T}^{2}\right)^{\frac{1}{2}} h_{E}^{-\frac{1}{2}}\left\|\vec{R}_{E}\right\|_{O, E} \\
+\left\|\vec{R}_{T}\right\|_{O, T} h_{E}^{\frac{1}{2}}\left\|\vec{R}_{E}\right\|_{O, E}
\end{gathered}
$$

Using (69) gives

$$
\int_{E} \vec{R}_{E} \cdot \vec{w}_{E} \leq C\left(\|\vec{e}\|_{J, T}^{2}+\|\varepsilon\|_{O, T}^{2}\right)^{\frac{1}{2}} h_{E}^{-\frac{1}{2}}\left\|\vec{R}_{E}\right\|_{\mathrm{O}, E}
$$

Using (49), then

$\int_{E} \vec{R}_{E} \cdot \vec{w}_{E}=\left\|\vec{R}_{E} b_{E}^{\frac{1}{2}}\right\|_{0, E}^{2} \geq c\left\|\vec{R}_{E}\right\|_{0, E}^{2}$,

and using (71), gives

$$
h_{E}\left\|\vec{R}_{E}\right\|_{0, \mathrm{E}}^{2} \leq \mathrm{C}\left(\|\overrightarrow{\mathrm{e}}\|_{J, T}^{2}+\|\varepsilon\|_{0, T}^{2}\right)
$$

Finally, combining (68), (69), (71) and (73) establishes the local lower bound.

B. The Local Poisson Problem Estimator.

The local Poisson problem estimator defined as:

$$
\begin{gathered}
\eta_{P}=\sqrt{\sum_{T \in T_{h}} \eta_{P, T}^{2}} \\
\eta_{P, T}^{2}=\left\|\vec{e}_{P, T}\right\|_{J, T}^{2}+\left\|\varepsilon_{P, T}\right\|_{0, T}^{2}
\end{gathered}
$$

Let

- $V_{T}=\left\{\vec{v} \in H^{1}(T): \vec{v} \cdot \vec{n}=0\right.$ on $\left.\partial \mathrm{T} \cap \partial \Omega\right\}$

- $A_{T}\left(\vec{e}_{P, T}, \vec{v}\right)=v \int_{T} \nabla \vec{e}_{P, T}: \nabla \vec{v}+\int_{\partial T} \vec{e}_{P, T}^{T} A \vec{v}$.

$\vec{e}_{P, T} \in V_{T}$ Satisfies the uncoupled Poisson problems

$$
A_{T}\left(\vec{e}_{P, T}, \vec{v}\right)=\left(\vec{R}_{T}, \vec{v}\right)_{T}-\sum_{E \in \varepsilon(T)}\left(\vec{R}_{E}, \vec{v}\right)_{E}
$$

for any $\vec{v} \in V_{T}$.

$$
\text { And } \quad \varepsilon_{P, T}=\nabla \cdot \vec{u}_{h} / T \text {. }
$$

Theorem 6.11. $\eta_{P, T}$ is equivalent to $\eta_{R, T}$ estimator:

$$
c \eta_{P, T} \leq \eta_{R, T} \leq C \eta_{P, T} \text {. }
$$

Proof. For the upper bound, we first let $\vec{w}_{T}=\vec{R}_{T} b_{T}\left(b_{T}\right.$ is an element interior bubble).

From (75),

$$
\begin{aligned}
\left(\vec{R}_{T}, \vec{w}_{T}\right)_{T}= & v\left(\nabla \vec{e}_{P, T}, \nabla \vec{w}_{T}\right)_{T} \\
& \leq v\left|\vec{e}_{P, T}\right|_{1, T}\left|\vec{w}_{T}\right|_{1, T}
\end{aligned}
$$

Using (48) we get

$$
\left(\vec{R}_{T}, \vec{w}_{T}\right)_{T} \leq C h_{T}^{-1}\left\|\vec{R}_{T}\right\|_{0, T}\left(\left\|\vec{e}_{P, T}\right\|_{J, T}^{2}+\left\|\varepsilon_{P, T}\right\|_{0, T}^{2}\right)^{\frac{1}{2}}
$$

In addition, from the inverse inequalities (47), $\left\|\vec{R}_{T}\right\|_{0, T}^{2} \leq$ C. $\left(\vec{R}_{T}, \vec{w}_{T}\right)_{T}$ And using (77), to get

$$
h_{T}^{2}\left\|\vec{R}_{T}\right\|_{0, T}^{2} \leq C\left(\left\|\vec{e}_{P, T}\right\|_{J, T}^{2}+\left\|\varepsilon_{P, T}\right\|_{0, T}^{2}\right)
$$

Next, we let $\vec{w}_{E}=\vec{R}_{E} b_{E}$ ( $b_{E}$ is an edge bubble function).

If $E \in \partial T \cap \varepsilon_{h, \Gamma}$, using (75), (78), (50) and (51) give

$$
\begin{aligned}
\left(\vec{R}_{E}, \vec{w}_{E}\right)_{E} & =-A_{T}\left(\vec{e}_{P, T}, \vec{w}_{E}\right)_{T}+\left(\vec{R}_{T}, \vec{w}_{E}\right)_{T} \\
& \leq\left\|\vec{e}_{P, T}\right\|_{J, T}\left\|\vec{w}_{E}\right\|_{J, T}+\left\|\vec{R}_{T}\right\|_{0, T}\left\|\vec{w}_{E}\right\|_{0, T} \\
& \leq C h_{E}^{-\frac{1}{2}}\left\|\vec{R}_{E}\right\|_{0, E}\left(\left\|\vec{e}_{P, T}\right\|_{J, T}^{2}+\left\|\varepsilon_{P, T}\right\|_{0, T}^{2}\right)^{\frac{1}{2}}
\end{aligned}
$$

if $E \in \partial T \cap \varepsilon_{h, \Omega}$,

See that the matrix $A$ defined just in $\Gamma$, then we can posed

$$
A=\mathrm{O} \text { in } \Omega-\Gamma \text {. }
$$

Using (75), (50), (51) and (78) give

$$
\begin{aligned}
&\left(\vec{R}_{E}, \vec{w}_{E}\right)_{E}=-v\left(\nabla \vec{e}_{P, T}, \nabla \vec{w}_{E}\right)_{T}+\left(\vec{R}_{T}, \vec{w}_{E}\right)_{T} \\
& \leq v\left|\vec{e}_{P, T}\right|_{1, T}\left|\vec{w}_{E}\right|_{1, T}+\left\|\vec{R}_{T}\right\|_{0, T}\left\|\vec{w}_{E}\right\|_{0, T} \\
& \leq C h_{E}^{-\frac{1}{2}}\left\|\vec{R}_{E}\right\|_{0, E}\left(\left\|\vec{e}_{P, T}\right\|_{J, T}^{2}+\left\|\varepsilon_{P, T}\right\|_{0, T}^{2}\right)^{\frac{1}{2}}
\end{aligned}
$$

Finally, for any $\mathrm{T} \in T_{h}$ and any $E \in \partial T$, we have

$$
\left(\vec{R}_{E}, \vec{w}_{E}\right)_{E} \leq C h_{E}^{-\frac{1}{2}}\left\|\vec{R}_{E}\right\|_{0, E}\left(\left\|\vec{e}_{P, T}\right\|_{J, T}^{2}+\left\|\varepsilon_{P, T}\right\|_{0, T}^{2}\right)^{\frac{1}{2}}
$$

From this result and the inverse inequalities (49), give

$$
h_{E}\left\|\vec{R}_{T}\right\|_{0, T}^{2} \leq C\left(\left\|\vec{e}_{P, T}\right\|_{J, T}^{2}+\left\|\varepsilon_{P, T}\right\|_{0, T}^{2}\right) .
$$

We have also $\left\|R_{T}\right\|_{0, T}=\left\|\nabla \cdot \vec{u}_{h}\right\|_{0, T}==\left\|\varepsilon_{P, T}\right\|_{0, T}$, then

$$
\left\|R_{T}\right\|_{0 . T}^{2} \leq\left(\left\|\vec{e}_{P, T}\right\|_{J, T}^{2}+\left\|\varepsilon_{P, T}\right\|_{0, T}^{2}\right)
$$

Combining (78), (79) and (80), establishes the upper bound in the equivalence relation.

For the lower, we need to use (65): 


$$
\begin{aligned}
\eta_{P, T} & =\left(\left\|\vec{e}_{P, T}\right\|_{J, T}^{2}+\left\|\varepsilon_{P, T}\right\|_{0, T}^{2}\right)^{\frac{1}{2}} \\
& \leq\left(\left\|\vec{e}_{P, T}\right\|_{J, T}+\left\|\varepsilon_{P, T}\right\|_{0, T}\right) \\
& \leq 2 \sup _{(\vec{v}, q) \in V_{T} \times L_{0}^{2}(T)} \frac{A_{T}\left(\vec{e}_{P, T}, \vec{v}\right)+d\left(\varepsilon_{P, T}, q\right)}{\|\vec{v}\|_{J, T}+\|q\|_{0, T}}
\end{aligned}
$$

Using (75) and (76) give

$$
\eta_{P, T} \leq 2 \sup _{(\vec{v}, q) \in V_{T} \times L_{0}^{2}(T)} \frac{\left(\vec{R}_{T}, \vec{v}\right)_{T}-\sum_{E \subset \partial T}\left(\vec{R}_{E}, \vec{v}\right)_{E}+\left(R_{T}, q\right)_{T}}{\|\vec{v}\|_{J, T}+\|q\|_{0, T}},
$$

then

$$
\eta_{P, T} \leq 2 \sup _{(\vec{v}, q) \in V_{T} \times L_{0}^{2}(T)} \frac{\left\|\vec{R}_{T}\right\|_{0, T}\|\vec{v}\|_{0, T}+\sum_{E \subset \partial T}\left\|\vec{R}_{E}\right\|\left\|_{0, E}\right\| \vec{v}\left\|_{0, E}+\right\| R_{T}\left\|_{0, T}\right\| q \|_{0, T}}{\|\vec{v}\|_{J, T}+\|q\|_{0, T}}
$$

Now, since $\vec{v}$ is zero at the four vertices of $\mathrm{T}$, a scaling argument and the usual trace theorem, see e.g. [15, Lemma 1.5], shows that $\vec{v}$ satisfies

$$
\begin{aligned}
\|\vec{v}\|_{0, E} & \leq C h_{E}^{\frac{1}{2}}|\vec{v}|_{1, T} \\
\|\vec{v}\|_{0, T} & \leq C h_{T}|\vec{v}|_{1, T}
\end{aligned}
$$

Combining these two inequalities with (82) immediately gives the lower bound in the equivalence relation.

Consequence 6.12. For any mixed finite element approximation (not necessarily inf-sup stable) defined on rectangular grids $T_{h}$, the residual estimator $\eta_{P}$ satisfies:

$$
\|\vec{e}\|_{J, \Omega}+\|\varepsilon\|_{0, \Omega} \leq C \eta_{P}
$$

Note that the constant $\mathrm{C}$ in the local lower bound independent of the domain.

\section{NUMERICAL SimULATION}

In this section some numerical results of calculations with mixed finite element Method and ADINA system will be presented. Using our solver, we run the flow over an obstacle [15] with a number of different model parameters.

Example: Flow over an obstacle. This is another classical problem. The domain is $\Omega$ and is associated with modelling flow in a rectangular channel with a square cylindrical obstruction. A Poiseuille profile is imposed on the Inflow boundary $(x=0 ;-1 \leq y \leq 1)$, and noflow (zero velocity) condition is imposed on the obstruction and the top and bottom walls. A Neumann condition is applied at the outflow boundary which automatically sets the mean outflow pressure to zero. $\Omega$ a disconnected rectangular region $(0,8) \times(-1,1)$ generated by deleting the square $(7 / 4,9 / 4) \times(-1 / 4,1 / 4)$.

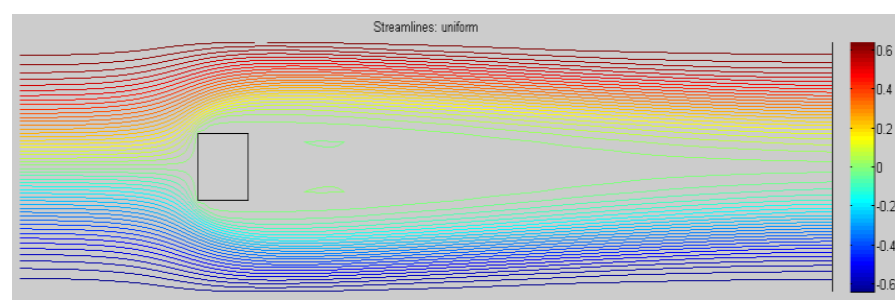

Fig.1. Equally distributed streamline plot associated with a $32 \times 80$ square grid $Q_{1}-P_{\mathrm{o}}$ approximation and $v=1 / 500^{\circ}$

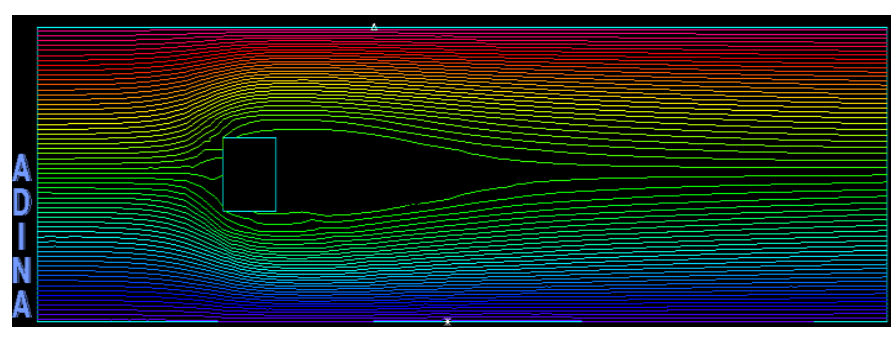

Fig.2. uniform streamline plot computed with ADINA System, associated with a $32 \times 80$ square grid and $v=1 / 500$

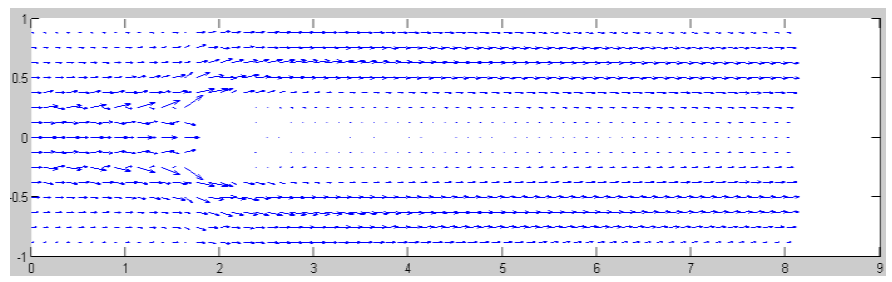

Fig.3. Velocity vectors solution by MFE with a $32 \times 80$ square grid and $v=1 / 500$.

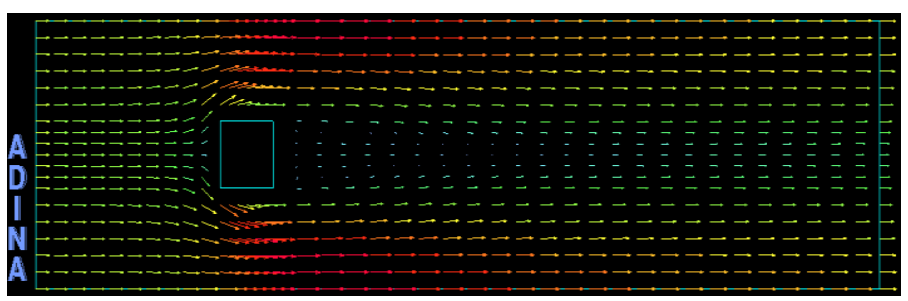

Fig.4. The solution computed with ADINA system. The plots show the velocity vectors solution with a $32 \times 80$ square grid and $v=1 / 500$.

The two solutions are therefore essentially identical. This is very good indication that my solver is implemented correctly.

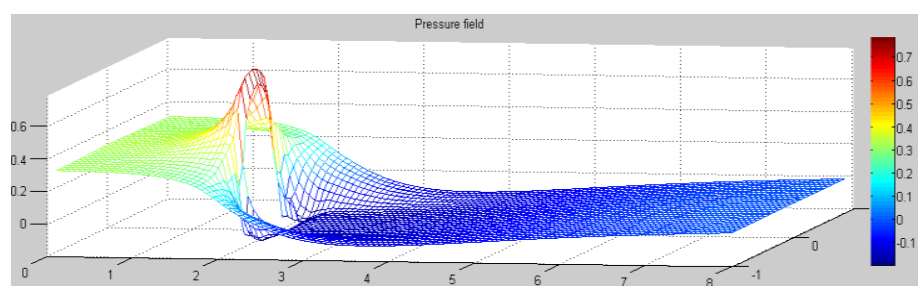

Fig.5. Pressure plot for the flow with a $32 \times 80$ square grid. 


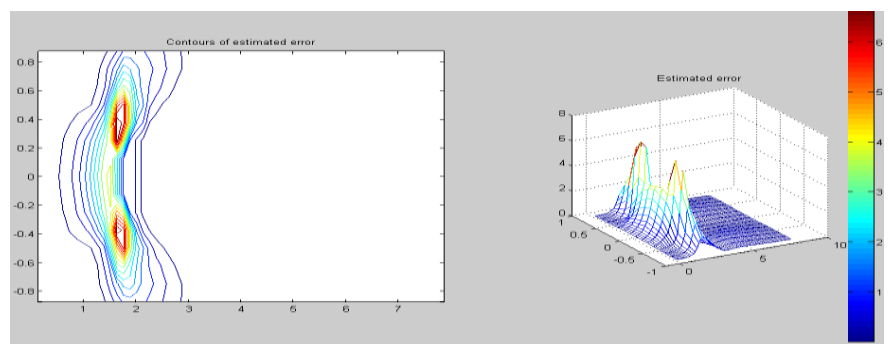

Fig.6. Estimated error $\eta_{R, T}$ associated with $32 \times 80$ square grid and $Q_{1}-P_{0}$ approximation

TABLE1. The local Poisson problem error estimator Flow over TABLE I. an obstacle with Reynolds number $\mathrm{Re}=1000$.

$\left\|\nabla \cdot \vec{u}_{h}\right\|_{\Omega}$ estimated velocity divergence error.

\begin{tabular}{l|l|c|} 
Grid & $\left\|\nabla \cdot \vec{u}_{h}\right\|_{0, \Omega}$ & $\eta_{P}$ \\
\hline $8 \times 20$ & $5.892389 \mathrm{e}-001$ & $3.210243 \mathrm{e}+001$ \\
\hline $16 \times 40$ & $1.101191 \mathrm{e}-001$ & $6.039434 \mathrm{e}+000$ \\
\hline $32 \times 80$ & $3.707139 \mathrm{e}-002$ & $2.802914 \mathrm{e}+000$ \\
\hline $64 \times 160$ & $1.160002 \mathrm{e}-002$ & $1.484983 \mathrm{e}+000$ \\
\hline
\end{tabular}

TABLE II. A residual error estimator for Flow over an obstacle with Reynolds number $\mathrm{Re}=1000$.

\begin{tabular}{l|l|} 
Grid & $\eta_{R}$ \\
\hline $8 \times 20$ & $9,309704 \mathrm{e}+00$ \\
\hline $16 \times 40$ & $1,727278 \mathrm{e}+000$ \\
\hline $32 \times 80$ & $8,156479 \mathrm{e}-001$ \\
\hline $64 \times 160$ & $4.261901 \mathrm{e}-001$ \\
\hline
\end{tabular}

\section{CONCLUSION}

We were interested in this work in the numeric solution for two dimensional partial differential equations modelling (or arising from) model steady incompressible fluid flow. It includes algorithms for discretization by mixed finite element methods and a posteriori error estimation of the computed solutions. Our results agree with Adina system.
Numerical results are presented to see the performance of the method, and seem to be interesting by comparing them with other recent results.

\section{ACKNOWLEDGMENT}

The authors would like to express their sincere thanks for the referee for his/her helpful suggestions.

\section{References}

[1] Alexandre Ern, Aide-mémoire Eléments Finis, Dunod, Paris, 2005.

[2] P.A. Raviart, J. Thomas, Introduction l'analyse numérique des équations aux dérivées partielles, Masson, Paris, 1983.

[3] E. Creuse, G. Kunert, S. Nicaise, A posteriori error estimation for the Stokes problem: Anisotropic and isotropic discretizations, M3AS, Vol.14, 2004, pp. 1297-1341.

[4] P. Clement, Approximation by finite element functions using local regularization, RAIRO. Anal. Numer, Vol.2, 1975, pp. 77-84.

[5] T. J. Oden,W.Wu, and M. Ainsworth. An a posteriori error estimate for finite element approximations of the Navier-Stokes equations, Comput. Methods Appl. Mech. Engrg, Vol.111, 1994, pp. 185-202.

[6] V. Girault and P.A. Raviart, Finite Element Approximation of the Navier-Stokes Equations, Springer-Verlag, Berlin Heiderlberg New York, 1981.

[7] A. Elakkad, A. Elkhalfi, N. Guessous. A mixed finite element method for Navier-Stokes equations. J. Appl. Math. \& Informatics, Vol.28, No.5-6, 2010, pp. 1331-1345.

[8] R. Verfurth, A Review of A Posteriori Error Estimation and Adaptive Mesh-Refinement Techniques, Wiley-Teubner, Chichester, 1996.

[9] M. Ainsworth and J. Oden. A Posteriori Error Estimation in Finite Element Analysis. Wiley, New York, [264, 266, 330, 334, 335], 2000.

[10] M. Ainsworth, J. Oden, A posteriori error estimates for Stokes' and Oseen's equations, SIAM J. Numer. Anal, Vol. 34, 1997, pp. 228-245.

[11] R. E. Bank, B. Welfert, A posteriori error estimates for the Stokes problem, SIAM J. Numer. Anal, Vol.28, 1991, pp. 591-623.

[12] C. Carstensen,S.A. Funken. A posteriori error control in low-order finite element discretizations of incompressible stationary flow problems. Math. Comp., Vol.70, 2001, pp. 1353-1381.

[13] D. Kay, D. Silvester, A posteriori error estimation for stabilized mixed approximations of the Stokes equations, SIAM J. Sci. Comput, Vol.21, 1999, pp. 1321-1336.

[14] R. Verfurth, A posteriori error estimators for the Stokes equations, Numer. Math, Vol.55, 1989, pp. 309-325.

[15] H. Elman, D. Silvester, A. Wathen, Finite Elements and Fast Iterative Solvers: with Applications in Incompressible Fluid Dynamics, Oxford University Press, Oxford, 2005

[16] V. John. Residual a posteriori error estimates for two-level finite element methods for the Navier-Stokes equations, App. Numer. Math., Vol.37, 2001, PP. 503-518.

[17] D. Acheson, Elementary Fluid Dynamics, Oxford University Press, Oxford, 1990. 